Introduction/Background* The clinical presentation of advanced USC may overlap significantly with that of serous carcinoma of tubo-ovarian origin. Differentiation facilitates patient counselling; guides choice of imaging; accurately stages; and fully informs MDT discussion - bearing in mind primary surgery as the treatment of choice for the less chemo-sensitive USC.

Methodology Cases of serous carcinoma initially presumed of tubo-ovarian origin, but confirmed on final histopathology as USC, were identified and patient care records reviewed retrospectively.

Result(s)*

Three cases were identified Mean age 75.7years. All presented with pain, distension but no post-menopausal bleeding. Each had an elevated CA125. Initial CT reported disseminated disease. Image-guided biopsy reported serous carcinoma. All three specimens were positive for p16, p53 and CA125; but only two WT1 positive (with focal/weak pattern) and one WT1 negative. After MDT discussion, all patients underwent neoadjuvant chemotherapy for presumed stage IIIc/IV tubo-ovarian serous carcinoma. Delayed primary surgery achieved R0 in all cases. Whilst final histopathology confirmed serous carcinoma, the diagnosis was amended to USC in each - arising from an endometrial polyp (mean size $16 \mathrm{~mm}$ ) in all three women.

Conclusion* Whilst several immunohistochemical markers are universal between the tumour types, the literature suggests

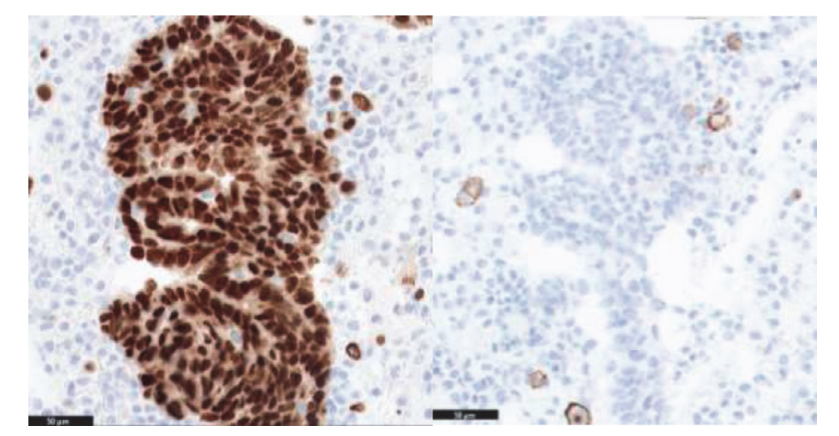

Abstract 552 Figure 1 Diffuse strong WT1 staining of tubo-ovarian HGSC v focal weak WT1 staining of USC that the majority $(94.7 \%)$ of serous carcinomas of tubo-ovarian origin exhibit positive WT1; compared to only $10-20 \%$ of USC. Further distinction arises in the pattern of WT1 positivity - strong/diffuse in tubo-ovarian versus focal/weak in the cross-over USC group. This correlates with our case series. We suggest that in disseminated serous carcinoma with negative WT1 or focal/weak positivity pre-operative hysteroscopy/ biopsy is considered. Whilst this may not always alter management, for patients on the border of suitability for primary surgery a preparedness to exert maximal surgical effort may be favoured on identifying USC.

\section{WHY PREFER DIFFUSION MRI OVER CT IN DIAGNOSTICS, STAGING AND DETECTION OF RECURRENCE IN OVARIAN CANCER PATIENTS? LNTERESTING ILLUSTRATIVE CASES}

${ }^{1} \mathrm{~K}$ Härmä*${ }^{2}{ }^{2} \mathrm{~S}$ Imboden, ${ }^{2} \mathrm{M}$ Mueller, ${ }^{1} \mathrm{~J}$ Heverhagen. ${ }^{1} / \mathrm{n}$ sel Gruppe $A G$, Bern University Hospital, University of Bern, Dep. of Diagnostic, Interventional and Pediatric Radiology, DIPR, Bern, Switzerland; ${ }^{2}$ Insel Gruppe AG, University of Bern, Department of Gynecology and Obstetrics Inselspital Bern, Bern, Switzerland

\subsection{6/ijgc-2021-ESGO.427}

Introduction/Background* Correct pre-operative staging and operability assessment is crucial for ovarian cancer (OC) patients' treatment decision. Diffusion weighted MRI (DWMRI) is a functional imaging technique, which is sensitive e.g. in detecting peritoneal, diaphragmal, liver capsular and bowel serosal carcinomatosis and has potential in the future to guide therapy assessments in personalized direction. Yet, DW-MRI is seldom used in daily clinical routine in OC.

Methodology We informed the gynecologist oncologist colleagues in our ESGO certified university hospital tumor center about the 3-Tesla whole-body DW-MRI modality, possible for OC. Until now, 25 patients are referred and included to the study. Acquisition time from the neck to symphysis requires 30 minutes and the protocol does not include contrast medium. Six women were referred because of suspicion on chemical or symptomatic recurrence (REC), 10 for the followup stating (F-up) after cancer therapy and six for primary

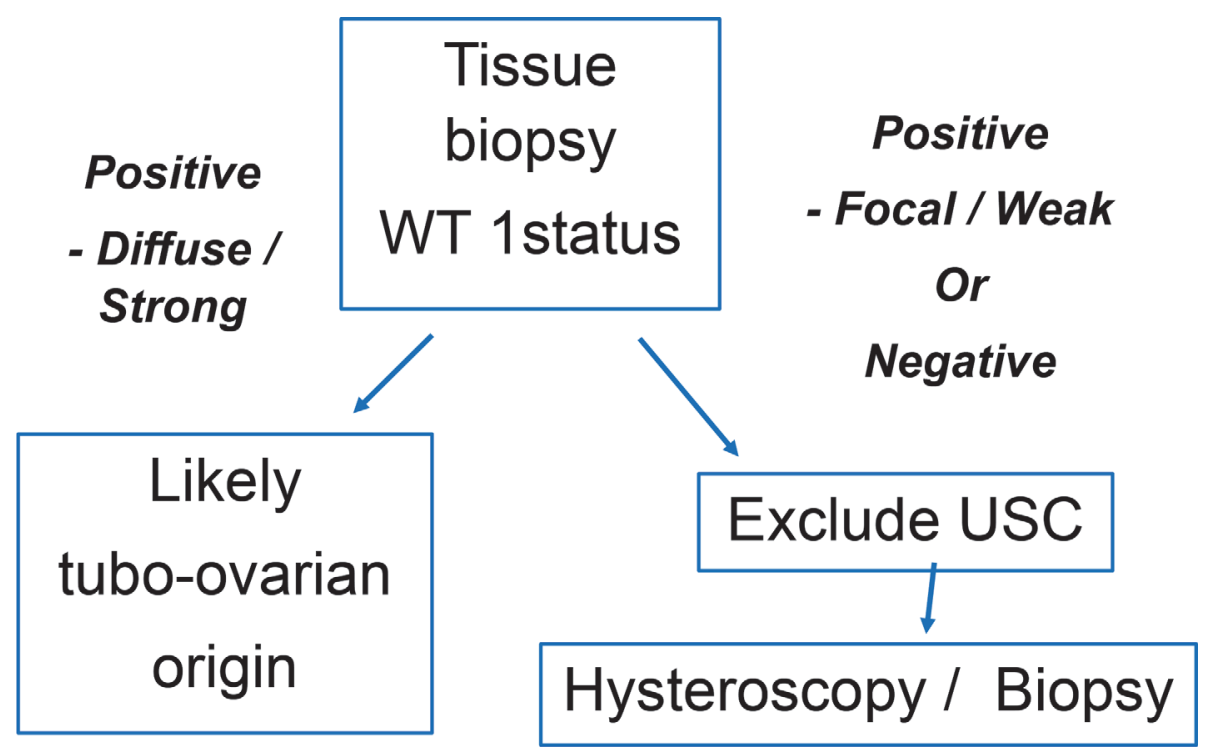

Abstract 552 Figure 2 Suggested pre-operative algorithm to distinguish USC v HGSC of tubo-ovarian origin 


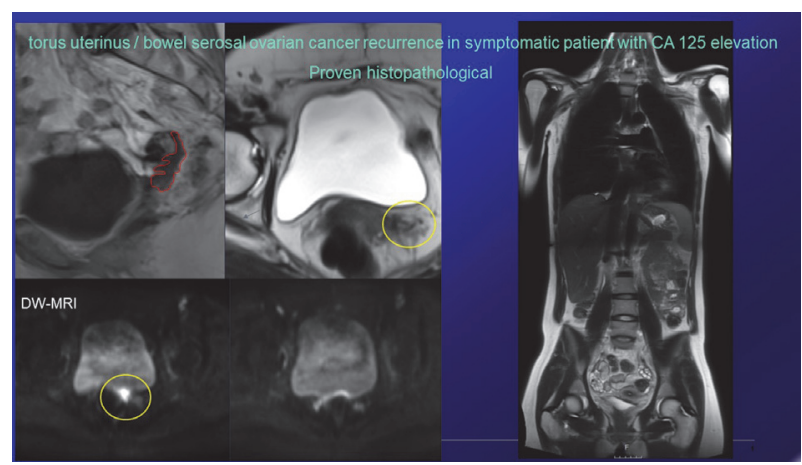

Abstract 554 Figure 1

tumor detection (Tu-Det). Furthermore, three patients were imaged having uterine, cervical and gestational breast cancer. Result(s)* 5/6 (REC), 5/10 (F-up), 4/6 (Tu-Det) showed pathologic diffusion restricted MR-graphic findings. Even MRgraphic only faint $\mathrm{T} 2 \mathrm{w}$ but diffusion restricted bright lesions were verified to be carcinomatous intraoperative/histopathological. Apparent diffusion coefficient (ADC) values were calculated. Primary and metastatic tumor sites were reported. When operated, the MR-graphic findings were correlated with intra-operative and histopathological findings.

Conclusion* DW-MRI without contrast media is a feasible and sensitive tool in OC primary diagnostics, staging and in detecting recurrence. Change is difficult, but sometimes necessary, as also former studies of this topic show. Larger multicenter studies are required in order to investigate the impact of DW-MRI for therapy management and overall survival.

\section{NON-BRCA1/2 PATHOGENIC/LIKELY PATHOGENIC VARIANTS DETECTED IN $2.6 \%$ OF PATIENTS WITH OVARIAN, FALLOPIAN TUBE OR PRIMARY PERITONEAL CANCER}

\footnotetext{
${ }^{1}$ VA Mesaric*, ${ }^{1} \mathrm{~K}$ Drusany Staric, ${ }^{2} \mathrm{~S}$ Hotujec, ${ }^{2} \mathrm{~K}$ Strojnik, ${ }^{2} \mathrm{~A}$ Blatnik, ${ }^{2} \mathrm{M}$ Banjac, ${ }^{3} \mathrm{E}$ Skof, ${ }^{4} \mathrm{~V}$ Stegel, ${ }^{4} \mathrm{~S}$ Novaković, ${ }^{2} \mathrm{M}$ Krajc. ${ }^{1}$ University Medical Center Ljubljana, Department of Gynaecology, Ljubljana, Slovenia; ${ }^{2}$ Institute of Oncology Ljubljana, Cancer Genetics Clinic, Ljubljana, Slovenia; 3 Institute of Oncology Ljubljana, Division of Medical Oncology, Ljubljana, Slovenia; ${ }^{4}$ Institute of Oncology Ljubljana, Department of Molecular Diagnostics, Ljubljana, Slovenia
}

\subsection{6/ijgc-2021-ESGO.428}

Introduction/Background* In Slovenia, ovarian cancer is diagnosed in approximately 160 women per year. The majority of patients are diagnosed with advanced disease and the survival rate is poor. $20-30 \%$ of cases can be attributed to germline pathogenic/likely pathogenic variants $(\mathrm{P} / \mathrm{LPV})$ in genes, associated with hereditary breast and ovarian cancer syndrome (HBOC). P/LPV in BRCA1/2 genes are the most prevalent. With the development of new methods for genetic testing, such as next generation sequencing (NGS), associations between non-BRCA genes and HBOCare being discovered.

Methodology We analyzed genetic results of patients with the diagnosis of ovarian, fallopian tube or primary peritoneal cancer, who were concecutively referred for genetic assessment at our institution in the period between September 2014 and April 2021. Since September 2014 all such patients are routinely tested with NGS with a panel (TruSight Cancer panel

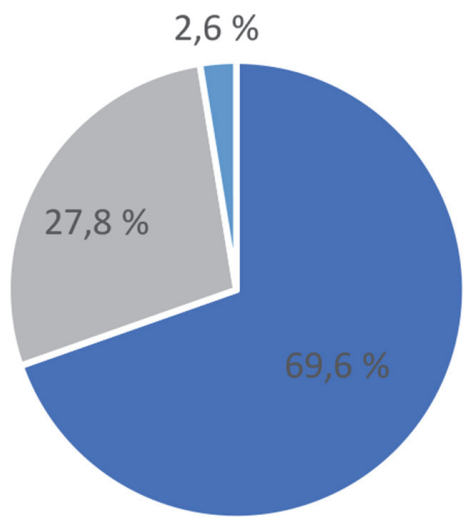

\section{- Negative - BRCA1/2 " Other than BRCA1/2}

Abstract 558 Figure 1 Results of genetic testing of patients with ovarian, fallopian tube or primary peritoneal cancer

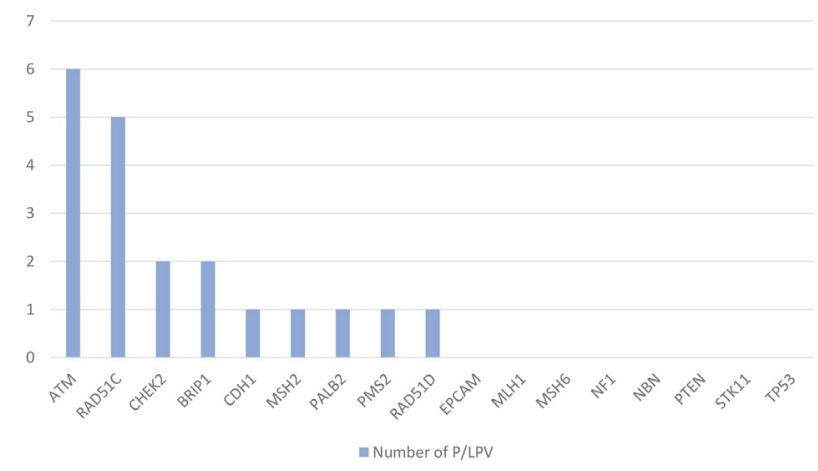

Abstract 558 Figure 2 Non-BRCA1/2 mutations in patients with ovarian, fallopian tube or primary peritoneal cancer, tested with our HBOC panel

or TruSight Hereditary Cancer panel) of 19 HBOC genes. In some cases, an analysis of tumor tissue was also performed.

Result(s)* During the observation period, 744 patients were tested for germline $\mathrm{P} / \mathrm{LPV}$ in HBOC gene panel. P/LPV was found in 226/744 (30.4\%) of tested patients. 207/744 (27.8\%) of patients had P/LPV in BRCA1/2. Non-BRCA P/LPVs were found in 19 patients (2.6\%), one patient had a P/LPV in both $A T M$ and $R A D 51 C$ genes (figure 1). The most common $\mathrm{P} /$ LPVs in non-BRCA genes were detected in ATM gene, and were diagnosed in 6 patients (figure 2). The results of tumor tissue testing were available for 7/19 nonBRCA-positive patients and in 1 biallelic PALB2 inactivation was found.

Conclusion* Among 744 consecutively tested patients with ovarian, fallopian tube or primary peritoneal cancer, we detected a high $\mathrm{P} / \mathrm{LPV}$ rate $(30.4 \%) .27 .8 \%$ of all tested patients were diagnosed with $B R C A 1 / 2$ P/LPVs. P/LPVs in other than $B R C A 1 / 2$ genes were detected less frequently (2.6\%). It is, however, important to be aware that P/LPVs in genes other than BRCA1/2can be detected in patients with ovarian cancer and that these women may also benefit from targeted treatment, preventive measures and further cascade testing in the family. Tumor testing may also reveal new treatment targets and help explain carcinogenesis in these patients. 